\title{
Time-dependent quantum models of the dynamics of neutron transfer reactions near the barrier
}

\author{
Viacheslav Samarin \\ Flerov Laboratory of Nuclear Reactions, JINR, 141980 Dubna, Moscow Region, Russia
}

\begin{abstract}
Time-dependent valence neutron wave functions were calculated for nucleus-nucleus collisions ${ }^{6} \mathrm{He}+{ }^{197} \mathrm{Au},{ }^{18} \mathrm{O}+{ }^{58} \mathrm{Ni},{ }^{48} \mathrm{Ca}+{ }^{18} \mathrm{O},{ }^{40,48} \mathrm{Ca}+{ }^{238} \mathrm{U}$. Probabilities of the neutron transfer and near-barrier population of two-center levels were calculated. The energy dependence of the neutron transfer cross section in the ${ }^{6} \mathrm{He}+{ }^{197} \mathrm{Au}$ reaction has been explained.
\end{abstract}

\section{Introduction}

The numerical solving of the time-dependent Schrödinger equation (TDSE) provides new possibilities for theoretical study of transfer reactions and the first capture stage of fusion reactions [1]. In this study the spin-orbital interaction [2], two-center states [3-6], the Pauli's exclusion principle [7] and the shell structure of deformed nuclei $[8,9]$ were taken into consideration.

\section{The solving of a time-dependent Schrödinger equation}

For the description of transfer reactions and the first capture stage of fusion reactions we used the approximation of the independent external (valence) neutrons. The two-component spinor wave function of each neutron changes according to the time-dependent Schrödinger equation (TDSE) with the spin-orbital interaction

$$
i \hbar-\left(\begin{array}{l}
1 \\
2
\end{array}\right)=\left\{\frac{\hbar^{2}}{2 m}+V(\mathbf{r}, t)+V_{L S}(\mathbf{r}, t)\right\}\left(\begin{array}{l}
1 \\
2
\end{array}\right) .
$$

The potential energy of a neutron with a vector radius $\mathbf{r}$ up to the contact with the colliding nuclei surfaces is the sum of the energies of its interaction with each nucleus

$$
\left.V(\mathbf{r}, t)=V_{1}\left(\begin{array}{ll}
\mathbf{r} & \mathbf{r}_{1}(t)
\end{array}\right)\right)+V_{2}\left(\begin{array}{ll}
\mathbf{r} & \mathbf{r}_{2}(t)
\end{array}\right) .
$$

Radii of nuclei centers $\mathbf{r}_{1}(t), \mathbf{r}_{2}(t)$ are determined from equations of the classical mechanics for colliding nuclei. The operator of the spin-orbital interaction is

$$
V_{L S}=\frac{b}{2 \hbar} \mathbf{y}[(V) \mathbf{p}] .
$$

Here, $\mathbf{p}$ is the momentum operator, $\mathbf{y}$ are Pauli matrices, the factor $b$ is

$$
b=R_{0}^{2} \frac{\hbar^{2}}{2 m^{2} R_{0}^{2} c^{2}}=0.022 R_{0}^{2},
$$

is the phenomenological dimensionless constant, $\sim 40, c$ is the velocity of light and $R_{0}=1 \mathrm{fm}$. On the grid coordinates the splitting method $[10,11]$ results in the difference scheme of the second order accuracy. Here, the equation (1) is solved iteratively in time with the fast complex Fourier transform [11] on a spatial grid with a plane of symmetry (the collision plane). The lattice spacing in the TDSE method is $0.2 \mathrm{fm}$, which is substantially smaller than $0.8 \mathrm{fm}$ in a typical timedependent Hartree-Fock (TDHF) calculation [12]. The colliding nuclei are enclosed in a box of typical dimensions $\begin{array}{lllll}48 & 24 & 24 & \mathrm{fm}^{3} & \text { for central collisions and }\end{array}$

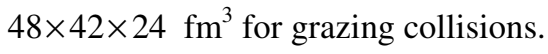

\section{The neutrons rearrangement and transfer in low-energy nuclear reactions with spherical nuclei}

\subsection{The neutrons stripping in the reaction ${ }^{6} \mathrm{He}+{ }^{197} \mathrm{Au}$}

TDSE (1) was solved for valence halo neutrons $1 p_{3 / 2}^{2}$ of the spherical nucleus ${ }^{6} \mathrm{He}$ at their collisions with a ${ }^{197} \mathrm{Au}$ nucleus. The typical change of the total sub-shell probability density

$$
(\mathbf{r}, t)=\sum_{=j}^{j}\left(\left|{ }_{1}\right|^{2}+\left.\left.\right|_{2}\right|^{2}\right)
$$

\footnotetext{
${ }^{\mathrm{a}}$ Corresponding author: samarin@jinr.ru
} 
is illustrated in Fig. 1. Here $=1 / 2,3 / 2, \ldots$ is the total angular momentum projection onto the axis connecting the centers of colliding nuclei (the inter-nuclear axis). (a)

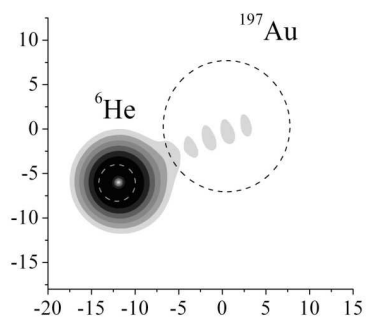

(c)

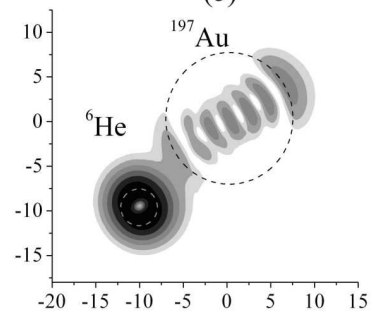

(b)

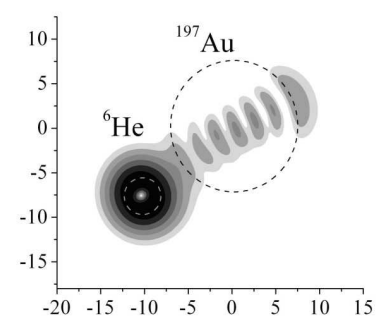

(d)

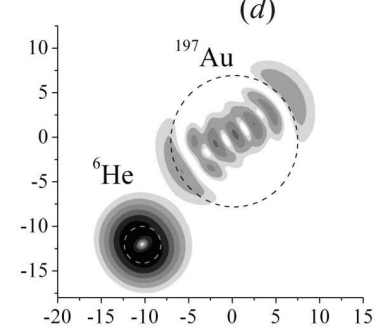

Figure 1. The change in the probability density of the valence $1 p_{3 / 2}^{2}$ neutrons of the ${ }^{6} \mathrm{He}$ nucleus during a collision with the ${ }^{197} \mathrm{Au}$ nucleus at an energy in the centre of mass system $E=19$ $\mathrm{MeV}$, a scale factor is $1 \mathrm{fm}$, and radii of the circumferences equal to radii of the nuclei. The course of time corresponds to panels from $(a)$ to $(d)$.

It is found, that the nucleons rearrangement and transfer are most probable for small values of full neutron angular moment projection on an inter-nuclear axis at the closed approach of nuclei. The small lattice spacing $(0.2 \mathrm{fm})$ results in correct calculations of the spatial structure of the spinor wave function. The presence of a stable structure in Fig. 1 indicates to the formation of two centre (molecular) states of external neutrons. The neutron cloud in this case covers both the nuclei, and an appropriate integral can serve as a measure of the probability of the neutron transfer from one nucleus to another. Results of the cross section calculation for the formation of the ${ }^{198} \mathrm{Au}$ isotope in the ${ }^{6} \mathrm{He}+{ }^{197} \mathrm{Au}$ reaction [13] agree satisfactorily with the experimental data [14] near the barrier (Fig. 2).

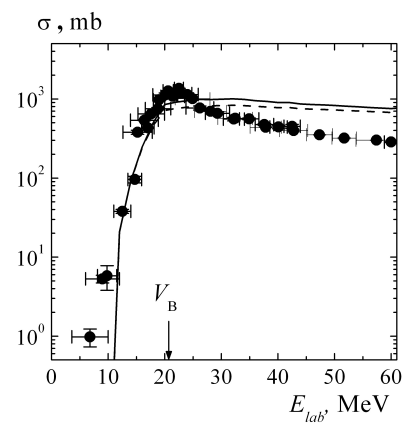

Figure 2. The energy dependence of the cross section for the formation of the ${ }^{198} \mathrm{Au}$ isotope in the ${ }^{6} \mathrm{He}+{ }^{197} \mathrm{Au}$ reaction. Dots represent the experimental data from [14]; the dashed line, calculations for the transfer of one neutron; the solid line, calculations for the transfer of one or two neutrons. $V_{\mathrm{B}}$ is the Coulomb barrier.
The ${ }^{198} \mathrm{Au}$ isotope can also be formed as a result of the transfer of two neutrons with subsequent evaporation of one of them. After the transfer of two neutrons to the high-lying levels of the Au nucleus, one neutron can fall to a lower level, transferring a part of the energy to the other neutron, which, as a result, abandons the $\mathrm{Au}$ nucleus. In our calculations, we used an approximation of the spherical shape conservation of the nuclear core (alpha-particle) of the ${ }^{6} \mathrm{He}$ and ${ }^{197} \mathrm{Au}$ nuclei during the collision. The difference between the experimental data and the calculation results at the sub-barrier energies of $E<V_{\mathrm{B}}$ could be due to possible deformations of the ${ }^{197} \mathrm{Au}$ nucleus under the influence of the nuclear force between this nucleus and the ${ }^{6} \mathrm{He}$ nucleus. The difference between the experimental data and the calculation results at the above-barrier energies of $E>V_{\mathrm{B}}$ could be due to the substantial changes in the states of the rest nucleons of colliding nuclei (except for the considered halo neutrons of the nucleus projectile) in inelastic and deeply inelastic processes.

\subsection{The neutrons rearrangement in the central collision ${ }^{18} \mathrm{O}+{ }^{58} \mathrm{Ni}$}

The typical shape of valence neutrons total probability density in system ${ }^{18} \mathrm{O}+{ }^{58} \mathrm{Ni}$ has a structure similar to two-center states [15] at a distance near the barrier (Fig. 3).

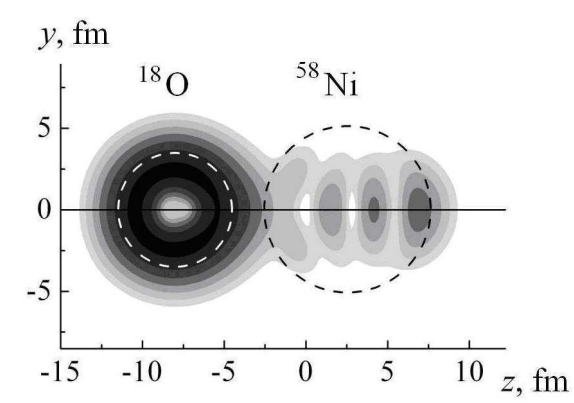

Figure 3. The probability density of valence $1 d_{5 / 2}^{2}$-neutrons of ${ }^{18} \mathrm{O}$ nucleus in the system ${ }^{18} \mathrm{O}+{ }^{58} \mathrm{Ni}$ near the barrier [15]

Two-center wave functions $(\mathbf{r}, R)$ and energies

$(R)$ of the valence neutron may be calculated in the two-center shell model by solving a stationary Schrödinger equation for the distance $R$ between the nuclei

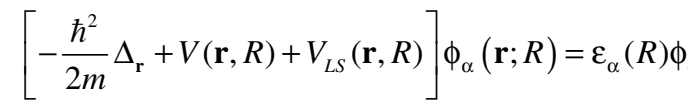

The equation (6) was solved by the method based on the series expansion of Bessel functions $[15,16]$. The total angular momentum projection is the quantum number of the two-center state $(R)={ }_{n},(R), \quad={ }_{n}$, . For the reaction ${ }^{18} \mathrm{O}+{ }^{58} \mathrm{Ni}$ some coefficients $a$ of the series expansion of the time-dependent neutron wave function $(\mathbf{r}, t)$ 


$$
(\mathbf{r}, t)=\sum a(R(t)) \quad(\mathbf{r} ; R(t))
$$

for the head-on nucleus-nucleus collision are plotted in Fig. 4.

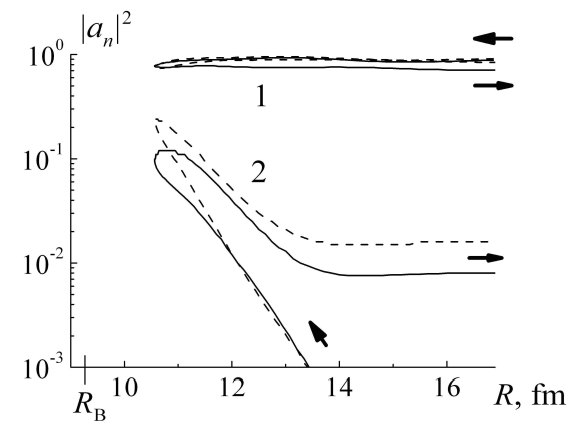

Figure 4. The population $\left|a_{n,}(R)\right|^{2}$ of two-center levels $1 d_{5 / 2}$ of ${ }^{18} \mathrm{O}$ (curves 1) and $1 g_{9 / 2}$ of ${ }^{58} \mathrm{Ni}$ (curves 2) for the total angular momentum projection on the inter-nuclear axis $\Omega=1 / 2$ (solid curves) and $\Omega=3 / 2$ (dashed curves) for $E_{\text {c.m. }}=30 \mathrm{MeV}$ [15], $R_{\mathrm{B}}$ is the Coulomb barrier radius.

The near barrier neutron transition with $Q>0$ from the $1 \mathrm{~d}_{5 / 2}\left({ }^{18} \mathrm{O}\right)$ state to the low-lied $1 \mathrm{~g}_{9 / 2}\left({ }^{58} \mathrm{Ni}\right)$ state is, in a certain sense, an "energy lift" for the two interacting nuclei [1]. The total barrier penetration probability and the fusion cross section may increase due to a gain in the relative motion energy in this case.

\subsection{The neutrons transfers and the Pauli's exclusion principle}

The Pauli's exclusion principle limits neutron transfers to occupied states. For two colliding nuclei with comparable values of the neutron separation energy we used few particles $(N=2,3)$ Slater determinants made up on the basis of time dependent wave functions for [7]

$$
{ }_{N}=\frac{1}{\sqrt{N !}} \operatorname{det}\left[\begin{array}{ccc}
{ }_{1}\left(\mathbf{r}_{1}, t\right) & \ldots & { }_{1}\left(\mathbf{r}_{N}, t\right) \\
\ldots & \ldots & \ldots \\
{ }_{N}\left(\mathbf{r}_{1}, t\right) & \ldots & { }_{N}\left(\mathbf{r}_{N}, t\right)
\end{array}\right] .
$$
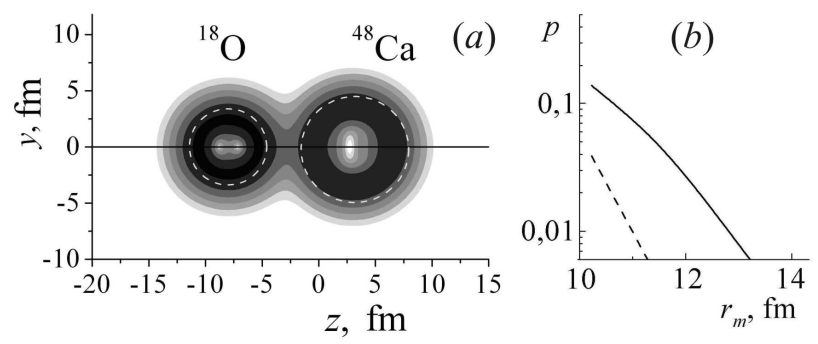

Figure 5. (a) The probability density of valence $1 d_{5 / 2}^{2}$-neutrons of ${ }^{18} \mathrm{O}$ nucleus and $1 f_{7 / 2}^{8}$-neutrons of ${ }^{48} \mathrm{Ca}$ nucleus in the vicinity of the Coulomb barrier [7]; $(b)$ the probability of neutrons stripping (solid curve) and pick-up (dashed curve) for ${ }^{18} \mathrm{O}$ at a central collision of ${ }^{18} \mathrm{O}+{ }^{48} \mathrm{Ca}$ as a function of the minimum value $r_{m}$ of the distance between nuclei centers.
A typical shape of valence neutrons total probability density (Fig. 5 a) in the reaction ${ }^{18} \mathrm{O}+{ }^{48} \mathrm{Ca}$ is similar to the results of the TDHF calculation [17]. The probabilities of neutron transfer are plotted in Fig. $5 b$, [7].

\section{Neutron transfers in reactions with a deformed nucleus ${ }^{238} \mathrm{U}$}

In this study the axially symmetric deformed WoodsSaxon potential

$$
V\left(\mathbf{r},{ }_{2},{ }_{4}\right)=V_{0}\left[1+\exp \left(\frac{r R\left(,_{2}, 4\right.}{a}\right)\right]^{1},
$$

is used in the shell model of a deformed nucleus ${ }^{238} \mathrm{U}$. We used the following values of deformation parameters ${ }_{2}=0.215,{ }_{4}=0.095$ for the nucleus of ${ }^{238} U$ [18]. The typical change of total sub-shell probability density for external (valence) neutrons $1 f_{7 / 2}^{8}$ in the reaction ${ }^{48} \mathrm{Ca}+{ }^{238} \mathrm{U}$ is illustrated in Fig. 6. The probability of neutron transfer is plotted in Fig. 7 [8, 9].
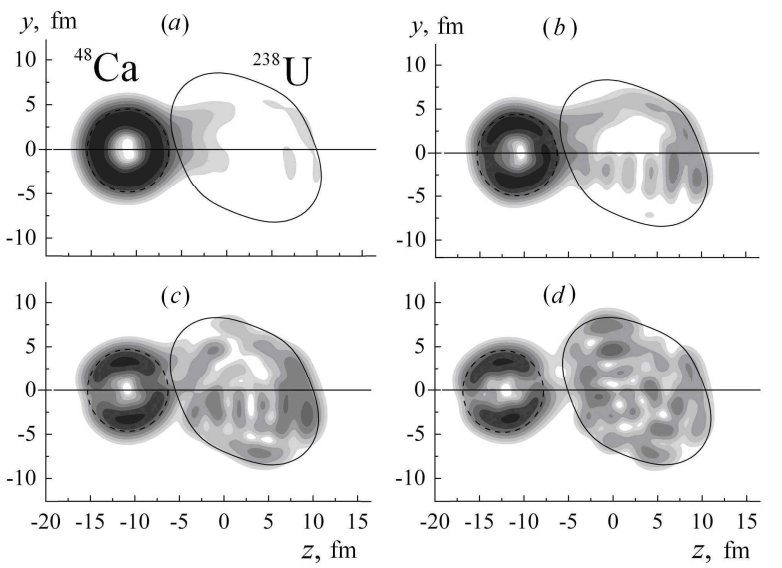

Figure 6. The change of probability density (5) of external neutrons of the $1 f_{7 / 2}^{8}$ shell of ${ }^{48} \mathrm{Ca}$ during the central collision with the ${ }^{238} \mathrm{U}$ at an energy in the center of mass system $E_{\text {c.m. }}=195 \mathrm{MeV}$. The angle between the symmetry axis of the deformed nucleus ${ }^{238} \mathrm{U}$ and the initial velocity of ${ }^{48} \mathrm{Ca}$ equals $45^{\circ}$. The course of time corresponds to panels from $(a)$ to $(d)$.

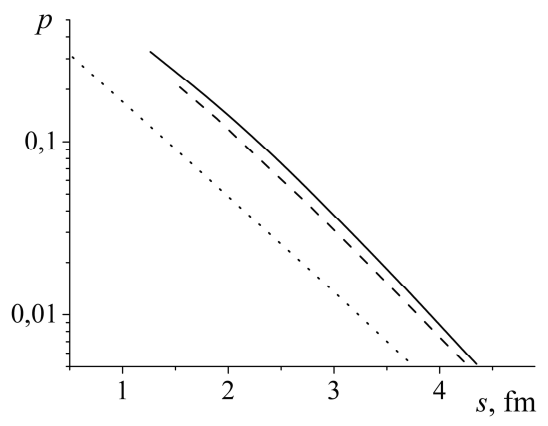

Figure 7. The probability of the neutron stripping in the reaction ${ }^{48} \mathrm{Ca}+{ }^{238} \mathrm{U}$ as a function of the minimum distance $s$ between nuclear surfaces. Angles between the symmetry axis of the deformed nucleus ${ }^{238} \mathrm{U}$ and the initial velocity of Ca nucleus equal $45^{\circ}$ (solid line), $90^{\circ}$ (dashed line) and 0 (dotted line). 
At the present time, most of calculations of the deformed nuclei shell structure are based on the NilssonStrutinsky method with the expansion in harmonicoscillator wave functions (see, for example, $[18,19])$. We used the above method based on the series expansion of Bessel functions $[15,16]$. The top upper occupied neutron energy level of deformed nucleus ${ }^{238} \mathrm{U}$ has the quantum number of the modulus of the total angular momentum projection $=5 / 2$ to the symmetry axis and the separation energy of $6.15 \mathrm{MeV}$ which is approximately equal to the experimental value. In the spherical nucleus limit ${ }_{2} \rightarrow 0,{ }_{4} \rightarrow 0$ this level becomes the level $1 j_{15 / 2}$ and we used its name for marking the corresponding level in the deformed nucleus. The typical change of the probability density for the valence neutron $1 j_{15 / 2}$ with $=5 / 2$ in the reaction ${ }^{40} \mathrm{Ca}+{ }^{238} \mathrm{U}$ is illustrated in Fig. 8. The probability of the neutron pick-up is plotted in Fig. $9[8,9]$.
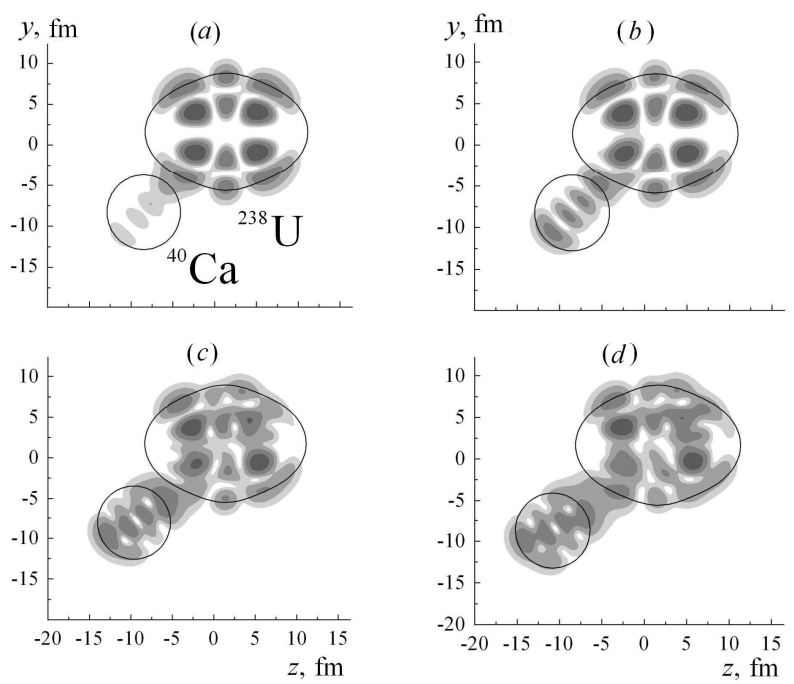

Figure 8. The probability density of the valence neutrons of ${ }^{238} \mathrm{U}$ for the initial state $1 j_{15 / 2}$ with the angular momentum projection on the symmetry axis $=5 / 2$ during the central collision with ${ }^{40} \mathrm{Ca}$, at the energy in the center of mass system $E_{\text {c.m. }}=195 \mathrm{MeV}$, the angle between the symmetry axis of deformed nucleus ${ }^{238} \mathrm{U}$ and the initial velocity of $\mathrm{Ca}$ nucleus equals $45^{\circ}$. The course of time corresponds to panels from (a) to $(d)$.

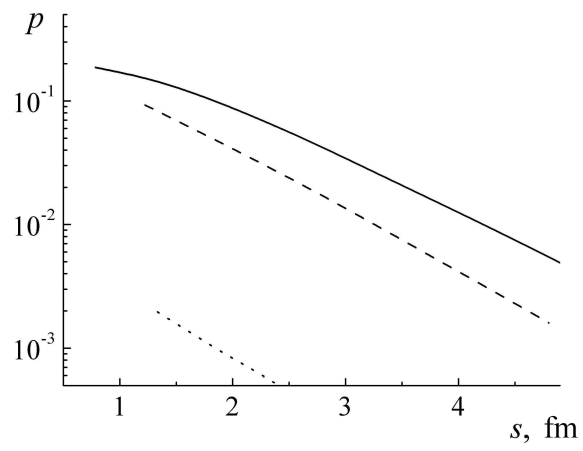

Figure 9. Probability of the neutron pick-up in the reaction ${ }^{40} \mathrm{Ca}+{ }^{238} \mathrm{U}$ as a function of the minimum distance $s$ between nuclear surfaces. Angles between the symmetry axis of the deformed nucleus ${ }^{238} \mathrm{U}$ and the initial velocity of $\mathrm{Ca}$ nucleus equal $45^{\circ}$ (solid line), $90^{\circ}$ (dashed line) and 0 (dotted line).

\section{Conclusions}

The proposed time-dependent quantum models based on the TDSE approach may be useful for the study of nucleons transfer reactions and the first capture stage of fusion reactions. They are easier than the TDHF method and allow more detailed calculation of the spatial structure of valence nucleons wave functions, than the TDHF method.

\section{Acknowledgments}

This work was partially supported by the Russian Foundation for Basic Research (RFBR), research project No 12-02-01325-a.

\section{References}

1. V. I. Zagrebaev, V. V. Samarin, W. Greiner. Phys. Rev. C 75, 035809 (2007)

2. V. V. Samarin and K. V. Samarin, Bull. Russ. Acad. Sci. Phys. 74, 567 (2010)

3. V. V. Samarin, Phys. of Atom. Nucl. 72, 1682 (2009)

4. V. V. Samarin, Phys. of Atom. Nucl. 73, 1416 (2010)

5. V. V. Samarin, Nucl. Phys. Atom. Ener. 14, 233 (2013)

6. V. V. Samarin, Phys. of Atom. Nucl. 77, 1124 (2014)

7. V. V. Samarin and K. V. Samarin, Proceedings of the VI International Symposium on Exotic Nuclei, EXON 2012 (Vladivostok, Russia, 1-6 October 2012) 111 (World Scientific Publishing, Singapore, 2013)

8. V. V. Samarin and K. V. Samarin, ibid, 203 (2013)

9. V. V. Samarin and K. V. Samarin, Bull. Russ. Acad. Sci. Phys. 78, 388 (2014)

10. M. E. Riley and B. Ritchie, Phys. Rev. A 59, 3544 (1999)

11. G. I. Marchuk, Methods of Computational Mathematics (Nauka, Moscow, 1980)

12. C. Golabek and C. Simenel, Phys. Rev. Lett. 103, 042701 (2009)

13. V. V. Samarin and K. V. Samarin, Bull. Russ. Acad. Sci. Phys. 76, 450 (2012)

14. A. Kulko et al., J. Phys. G 34, 2297 (2007)

15. V. V. Samarin, Phys. of Atom. Nucl. 77, N 12 (2014) (to be published)

16. V. V. Samarin, Phys. of Atom. Nucl. 73, 1416 (2010)

17. K. Washiyama and D. Lacroix, Phys. Rev. C. 78, 024610 (2008)

18. Nuclear Reaction Video (Low-Energy Nuclear Knowledge Base, FLNR, JINR, Dubna), http://nrv.jinr.ru/nrv/

19. K. Langanke and J. A. Maruhn, S. E. Koonin, Computational Nuclear Physics 1. Nuclear Structure. (Springer-Verlag, Germany, 1991) 